This paper appeared in the Proceedings of the ACM Workshop on Wireless Multimedia Networking and Performance Modeling, in conjunction with the 8th Int'l Symp. Modeling, Analysis and Simulation of Wireless and Mobile Systems (MSWiM 2005), Oct. 2005, Montreal, Quebec, Canada.

\title{
PARM: A Power-Aware Message Scheduling Algorithm for Real-Time Wireless Networks
}

\author{
Mohammed I Alghamdi \\ Department of Computer Science \\ New Mexico Institute of Mining and \\ Technology \\ 801 Leroy Place, P. O, BOX 2060 \\ Socorro, NM, 87801, U.S.A \\ 001-720-771-701 \\ alghamdi@nmt.edu
}

\author{
Tao Xie \\ Department of Computer Science \\ New Mexico Institute of Mining and \\ Technology \\ 801 Leroy Place, P.O. Box 2313, \\ Socorro, NM 87801, USA \\ 001-240- 784-0386 \\ xietao@cs.nmt.edu
}

\author{
Xiao Qin \\ Department of Computer Science \\ New Mexico Institute of Mining and \\ Technology \\ 801 Leroy Place, Socorro, NM, 87801 \\ 001-505-835-5902 \\ http://www.cs.nmt.edu/ xqin \\ xqin@cs.nmt.edu
}

\begin{abstract}
Real-time applications in wireless networks are emerging in multimedia product and design. However, conventional real-time message scheduling algorithms generally do not take energy efficiency into account when making scheduling decisions. In this paper, we address the issue of scheduling real-time messages in wireless networks subject to timing and power constraints. A novel message scheduling scheme, or PARM (Power-aware Realtime Message), is developed to generate optimal schedules that minimize both power consumption and the probability of missing deadlines for real-time messages. With a power-aware scheduling policy in place, the proposed PARM scheme is very energy efficient. In addition, we extended a power consumption model to calculate power consumption rates in accordance to message transmission rates. Experimental results show that PARM significantly improves the performance in terms of missed rate, energy efficiency, and overall performance over four baseline message scheduling schemes.
\end{abstract}

\section{Categories and Subject Descriptors \\ C.2 [Computer-Communication Networks]}

\section{General Terms}

Algorithms, Performance, Design

\section{Keywords}

Energy optimization, real-time systems, scheduling, wireless networks

Permission to make digital or hard copies of all or part of this work for personal or classroom use is granted without fee provided that copies are not made or distributed for profit or commercial advantage and that copies beat this notice and the full citation on the first page. To copy otherwise, or republish, to post on servers or to redistribute to lists, requires prior specific permission and/or a fee.

WMuNeP'05, October 13, 2005, Montreal, Quebec, Canada.

Copyright 2005 ACM 1-58113-000-0/00/004...\$5.00.

\section{INTRODUCTION}

Future generation wireless networks seamlessly provide users with multimedia and data services. There have been increasing effort to develop real-time applications in wireless networks. Advanced interactive multimedia applications are real-time in nature, and typical multimedia applications in wireless networks include video telephony, video games, and TV broadcasting. Real-time applications in wireless networks depend not only on the logical results of computation, but also on the time at which the results are produced [8][16][20].

Besides handling multimedia traffic like speech and video, challenges in improving wireless network capability include energy efficiency and different classes of data traffic. This is mainly because a large number of wireless devices are multimedia oriented, and most wireless devices have constraints on energy consumption, size, and bandwidth [2][4]. Recent studies show that battery performance improvement rate in terms of energy per unit size or weight is fairly slow, and energy consumption needed for communication will severely limit the functionality of wireless networks. Emerging multimedia applications with higher power needs make this problem even worse. Therefore, reducing power consumption of real-time traffic is an efficient approach to achieving long battery life for wireless networks supporting multimedia applications.

In recent years, processor energy conservation for real-time systems has been extensively studied. However, energy conservation in wireless networks using scheduling remains an open problem. Such energy conservation technologies become critical, because admission control and scheduling approaches are expected to significantly reduce energy consumption of real-time communication in wireless networks.

Scheduling algorithms play a key role in obtaining high performance in real-rime wireless data networks [3][9]. In addition to high performance, energy-aware message scheduling algorithms need to be developed to further improve energy efficiency of wireless networks. In this paper we propose an energy-aware scheduling algorithm or PARM for short, for wireless networks in the context of a future generation multimedia applications. The novelty of our approach lies in the fact that our message scheduling algorithm aims at meeting timing constrains 
and conserving power consumption for real-time wireless networks. To quantitatively evaluate our message scheduling algorithm, we built a simulation platform to compare our approach against four baseline algorithms that are unaware of energy efficiency.

The rest of this paper is organized as follows. Related work is discussed in Section 2. Section 3 describes the mathematical models. The new PARM algorithm is proposed in Section 4. Section 5 presents the simulation platform and experimental results of our scheduling scheme for wireless networks. Finally, Section 6 concludes the paper.

\section{RELATED WORK}

Some research has been geared towards real-time scheduling for real-time systems [1][6][16]. In practice, real-time scheduling algorithm generally falls into tow: static (off-line) [19] and dynamic (on-line) [14]. Lipari and Buttazzo proposed a framework for scheduling real-time multi-task applications in an open system [5]. Qin and Jiang developed an efficient faulttolerant scheduling algorithm for real-time tasks with precedence constraints [17]. Xie and Qin studied a security-aware dynamic scheduling algorithm for real-time applications running on grids [16]. Although these scheduling algorithms achieve high performance for real-time computation, they are not suitable for real-time wireless networks.

Recently increasing attention has been paid to energy-efficiency for wireless networks, because high energy-efficiency has become a baseline requirement. Jones examined the current state of the art in energy efficiency in the context of wireless networks and introduced new technologies that meet the energy-efficiency needs of wireless networks [2]. Stine and Veciana studied various methods to improve energy efficiency of centrally controlled wireless data networks [7]. Wang et al. proposed a new adaptive call admission control scheme that takes energy conservation in wireless networks into account [20]. However, the above energyefficiency techniques are not adequate for real-time applications due to the lack of ability to handle timing constraints.

Some work has been done to incorporate energy-efficiency into a variety of real-time applications. Havinga and Smit identified prominent problems of wireless multimedia networking and presented various solutions to improve energy efficiency for multimedia applications [10]. Lipari and Buuazzo proposed an approach to trading off quality of energy-efficiency to achieve required real-time performance [5]. Akkaya and Younis proposed an energy-aware QoS routing protocol used to find a least-cost, delay-constrained path for real-time data [21]. Our work is fundamentally different from the above approaches because our study is focused on the development of an energy-aware real-time scheduling algorithm, which can improve energy efficiency in addition to real-time requirements of task running on wireless networks.

\section{MODELS}

\subsection{Message scheduling architecture}

The scheduling architecture depicted in Figure 1 is mainly composed of an Energy Consumption Controller (ECC), an Admission Controller (AC) and an EDF scheduler (Earliest Deadline First Scheduling algorithm is applied). This architecture is deployed in a single base station, which receives incoming messages from $\mathrm{m}$ senders and delivers outgoing messages to $\mathrm{m}$ receivers. All the messages are independently submitted from $\mathrm{m}$ wireless terminals and their arrival times abide by Poisson distribution. The function of the admission controller is to determine if an incoming message can be accepted or not, whereas the energy consumption controller is intended to minimize the energy consumption of current messages residing in an accepted queue. The EDF scheduler transmits messages in accordance with their deadlines in a way that messages with the earliest deadlines are given the highest priority. If a real-time message's deadline cannot be guaranteed, the message will be dropped in the rejected queue.

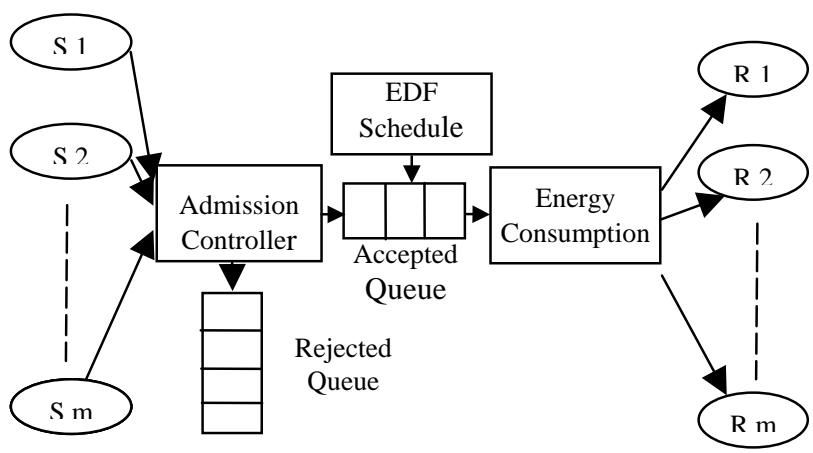

Figure 1. Architecture of the PARM strategy

\subsection{Modeling real-time messages}

Our real-time message model assumes that all messages have soft deadlines and all messages are independent of one another. In our system model, there exists a single base station handling real-time messages from $\mathrm{n}$ wireless terminals. Each message has a SenderID that indicates the identity of the message sender. Likewise, each message has a ReceiverID that hints where the message should be delivered. Messages arrive at the base station according to a Poisson process. Message $\mathrm{m}_{\mathrm{i}}$ is represented as a tuple $\left(a_{i}, f_{i}, \operatorname{sid}_{i}, \operatorname{rid}_{i}, s_{i}, d_{i}\right)$, where $a_{i}$ and $f_{i}$ are the arrival time and finish time of message $\mathrm{i}$. $\mathrm{sid}_{\mathrm{i}}$ and $\mathrm{rid}_{\mathrm{i}}$ represent the identity of the message sender and the identity of message receiver, respectively. $s d_{i}$ is the size of data embedded in the message $m_{i} . d_{i}$ is the soft deadline of message $m_{i}$. Note that given message $m_{i}$, all elements in the tuple above are known to the scheduler in advance. The transmission time for message $\mathrm{m}_{\mathrm{i}}$ can be computed by the following equation.

$$
t_{i}=s d_{i} / t r_{i},
$$

where $t_{i}$ is the transmission rate of message $m_{i} . M=\left\{m_{1}, m_{2}, \ldots\right.$ $\mathrm{m}_{\mathrm{n}}$ \} represents a set of real-time messages submitted to the single base station.

Our power-aware scheduler presented in the following section makes use of a function to measure the energy consumption of 
each admitted message. In particular, let $\mathrm{PC}_{\mathrm{i}}$ be the energy consumption of message $\mathrm{m}_{\mathrm{i}}$. It can be quantitatively computed by using Equation (2):

$$
P C_{i}=s d_{i} * f\left(t r_{i}\right)
$$

where $\mathrm{f}$ is a mapping function between transmission rate $\mathrm{tr}_{\mathrm{i}}$ and energy consumption rate $\mathrm{er}_{\mathrm{i}}$, which can be defined as $\mathrm{f}: \mathrm{tr}_{\mathrm{i}} \rightarrow \mathrm{er}_{\mathrm{i}}$ (see Figure 2).

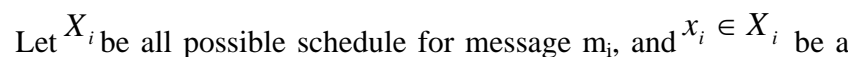
scheduling decision of $m_{i} \cdot x_{i}$ is a feasible schedule if deadline $d_{i}$ can be met, e.g., $f_{i} \leq d_{i}$. Given a real-time message $\mathrm{m}_{\mathrm{i}}$, the economical energy consumption of $\mathrm{M}_{\mathrm{i}}$ is expected to be minimized by the energy consumption controller (See Fig. 1) under the timing constraint:

$$
\operatorname{EPC}\left(X_{i}\right)=\min _{x_{i} \in X_{i}}\left\{P C\left(x_{i}\right)\right\} .
$$

An energy-aware scheduler strives to minimize the system's energy consumption, defined by the sum of the power consumption of all admitted messages. Let $X$ be all possible schedule for the entire message set $M$. Thus, the total energy consumption function needs to be minimized subject to timing constraints:

$$
\operatorname{TPC}(X)=\min _{X \in X}\left\{\sum_{i=1}^{n} y_{i} \operatorname{EPC}\left(X_{i}\right)\right\},
$$

where $n$ is the number of submitted messages, $y_{i}$ is set to 1 is message $m_{i}$ is admitted, and is set to 0 otherwise. Substituting Equation (3) into (4) yields the following energy consumption objective function.

$$
\operatorname{TPC}(X)=\min _{x \in X}\left\{\sum_{i=1}^{n} y_{i} \min _{x_{i} \in X_{i}}\left\{P C\left(x_{i}\right)\right\}\right\}
$$

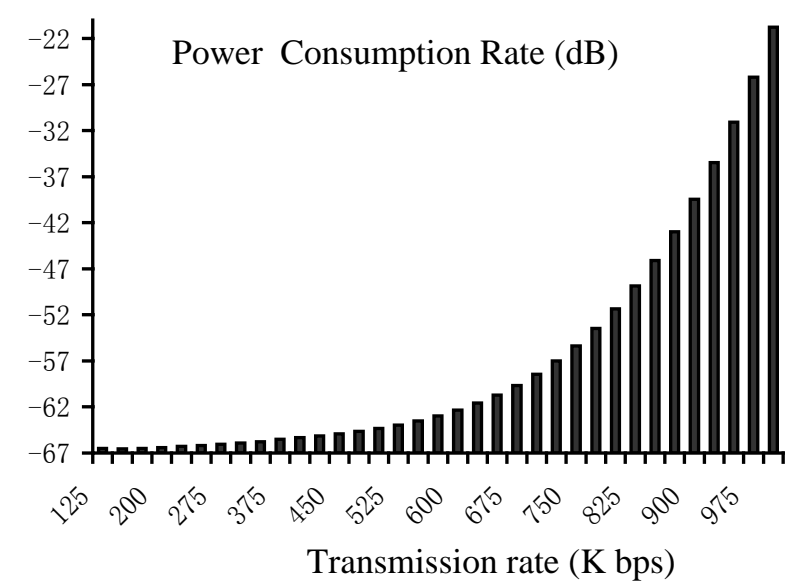

Figure 2. Energy consumption model

\subsection{Energy consumption model}

Figure 2 depicts the relationship between the power consumption rate and the message transmission rate. The vertical axis is the power consumption rate, whereas the horizontal axis is the message transmission rate measured in Kbps. We built the energy consumption model based on the empirical results reported in [20]. We extended the result in [20] using curve fitting so that we can quantitatively obtain corresponding power consumption when message transmission rate varies in the range between $125 \mathrm{kbps}$ and 1000kbps. Our PARM scheduling algorithm leverages the energy consumption model to calculate rates of power consumption according to message transmission rates determined by the energy consumption controller.

\section{THE SCHEDULING ALGORITHM}

Now we describe the PARM (Power-aware Real-time Message) scheduling algorithm, which integrates the earliest deadline first scheduling algorithm into the energy consumption heuristic scheme described in Section 3.

To facilitate the presentation of the proposed algorithm, we introduce the following property. The schedule of a message is feasible if the message can be delivered before its deadline. In other words, a message has a feasible schedule if it can be finished at the highest transmission rate. Formally, this fact can be express by the following property.

Property 1. If message $m_{i}$ has a feasible schedule, the following inequality must be satisfied:

$$
\begin{array}{r}
\text { es }\left(m_{i}\right)+t_{i}^{\text {min }} \leq d_{i}, \quad \text { under the condition stated below } \\
\forall m_{k} \in A Q, d_{k}>d_{i}: \operatorname{es}\left(m_{k}\right)+t_{k}^{\text {min }} \leq d_{k}
\end{array}
$$

where $A Q$ is the accepted queue, es $\left(m_{i}\right)$ is the earliest transmission start time of message $m_{i}$, and $t_{i}^{\min }$ is the minimal transmission time of message $m_{i}$ when its transmission rate is set to the highest value.

The condition enforced in Property 1 indicates that the transmission of $m_{i}$ results in no violation of any deadlines of messages that have been admitted to the base station.

The earliest start time es $\left(m_{i}\right)$ can be accurately computed by Equation (6).

$$
\operatorname{es}\left(m_{i}\right)=r+\sum_{m_{k} \in M} e_{k},
$$

where $r$ represents the remaining transmission time of a message currently transmitting by the base station., and $e_{k}$ is the transmission time of message $m_{k}$ whose deadline is earlier than that of $m_{i}$. Thus, the earliest transmission start time of $m_{i}$ is a sum of the remaining transmission time of the transmitting message and the transmission times of the messages with earlier deadlines.

The PARM algorithm is described in Figure 3. The goal of the PARM algorithm is to achieve low energy consumption while guaranteeing timing constraints of real-time messages submitted 
to a base station. More specifically, PARM manages to minimize energy consumption (measured by Equation 2) of each admitted message (see Step 16) while maintaining a reasonably high guarantee ratio, which is measured by the fraction of total arrived messages that are found to be schedulable (see Step 3).

Before optimizing the energy consumption of message $m_{i}$, PARM attempts to meet the real-time requirement of $m_{i}$ by calculating the earliest start time (use Equation 6) in Steps 2. Then, PARM performs a feasibility check, figuring out if $m_{i}$ can be successfully delivered before its deadline (see Step 3). In case where the deadline is missed, $m_{i}$ is rejected by the PARM algorithm (see Step 18).

\section{1. for each arrival message $m_{i}$ do}

2. Use Equation (6) to computer es $\left(m_{i}\right)$, the earliest transmissions start time of $m_{i}$;

3. if es $\left(m_{i}\right)+t_{i}^{\min } \leq d_{i}$ then (See Property 1)

4. while $t r_{i} \geq \min \left\{t r_{i}\right\}$ do

5. decrease transmission rate of $M_{i}$;

6. Use Equation (1) to calculate transmission rate of $M_{i}$;

7.(a) if es $\left(m_{i}\right)+t_{i}^{\text {min }}>d_{i}$ or

7.(b) $\forall m_{k} \in A Q, d_{k}>d_{i}: \operatorname{es}\left(m_{k}\right)+t_{k}^{\text {min }}>d_{k}$

(based on Property 1) then

8. $\quad$ decrease transmission rate of $m_{i}$; break;

9. $\quad$ end while

10. $t r_{i}^{r} \leftarrow t r_{i} ; / *$ Obtain the real transmission rate of $m_{i}$

$* /$

11. else

12. $t r_{i}^{r} \leftarrow 0$; /* Set the real transmission rate to 0 because $m_{i}$ has no feasible schedule */

13. end if

14. if $\operatorname{tr}_{i}^{r}>0$ then

15. $y_{i} \leftarrow 1 ;$; Accept message $m_{i}^{*}$

16. /* Optimize energy consumption of $m_{i}$, see Equation (3) */ Find a schedule for $m_{i}$, subject to: $\min _{x_{i} \in X_{i}}\left\{P C\left(x_{i}\right)\right\}$

17. else

18. $y_{i} \leftarrow 0$; /* Reject $m_{i}$, no feasible schedule is available */

19. end if

20.end for

\section{Figure 3. The PARM algorithm}

The energy consumption of message $m_{i}$ is optimized in Step 5, which decreases transmission rate of $m_{i}$ while satisfying the following two conditions. First, decreasing the transmission rate of $m_{i}$ will not miss the deadline of $m_{i}$. Second, the increment of the transmission time of $m_{i}$ due to the reduced transmission rate must not result in the missing deadlines of any previously admitted messages. These two conditions are respectively satisfied by Steps 7(a) and 7(b). Once Step 10 has dynamically decided the transmission rate of message $m_{i}$, step 16 can further minimize the energy consumption of $m_{i}$ by identifying the schedule for $m_{i}$ that provides the minimal transmission rate (see Step 16).

\section{EVALUATION RESULTS}

\subsection{Performance metrics and parameters}

To quantitatively evaluate the proposed PARM algorithm, we compared PARM against the following four baseline algorithms, namely, MIN-FIFO, MIN-EDF, MAX-FIFO, and MAX-EDF. The four baseline scheduling algorithms are briefly described below.

MIN-FIFO: Admitted messages are transmitted based on the First-In-Fist-Out policy. For each admitted message, the MINFIFO algorithm assigns the lowest bandwidth (e.g. $125 \mathrm{kbps}$ ), which leads to the lowest energy consumption.

MIN-EDF: Messages admitted by the system are delivered using the EDF policy. Similar to the MIN-FIFO algorithm, MIN-EDF transmits each admitted message using the lowest bandwidth.

MAX-FIFO: The First-In-Fist-Out policy is employed to schedule messages, and the highest bandwidth (e.g. $1000 \mathrm{kbps}$ ) is assigned to all admitted messages.

MAX-EDF: The EDF policy is used to schedule all admitted realtime messages. Regarding transmission rate assignment, MAX-

EDF allocates the highest bandwidth to admitted messages.

Table 1. Characteristics of System Parameters

\begin{tabular}{|l|l|}
\hline \multicolumn{1}{|c|}{ Parameter } & \multicolumn{1}{|c|}{ value } \\
\hline Networks bandwidth & $125-1000 \mathrm{Kbps}$ \\
Minimal deadline base & 100 Seconds \\
Maximal deadline base & 500 Seconds \\
Min data size & $500 \mathrm{~KB}$ \\
Max data size & $1000 \mathrm{~KB}$ \\
Arrival rate & $0.1-1.0$ No./Sec. \\
Number of messages & 10000 \\
\hline
\end{tabular}

Table 1 summarizes system parameters of a simulated wireless network used in our experiments. Although data size of messages, deadlines, and arrival rate are synthetically generated, we can evaluate impacts of these workload parameters on performance by controlling them as fundamental simulation parameters. The performance metrics by which we evaluate system performance include:

(1) Missed rate $(M R)$ : measured as a fraction of total submitted messages that missed their deadlines.

(2) Total power consumption (see Equation 4).

(3) Average power consumption. 
Overall system performance: defined by $1-w_{m r} \times M R-w_{p c} \times N P C$, where NPC is the normalized power consumption, $w_{m r}$ and $w_{p c}$ are two weights. In our experiments, $w_{m r}$ and $w_{p c}$ are set to 0.5 .

\subsection{Effect of arrival rate}

Figures 1-4 show the simulation results for these five algorithms. The deadlines are randomly generated in the range between 100 to 500 seconds, and the data size are randomly chosen in the range from 500 to 1000 Kbytes.

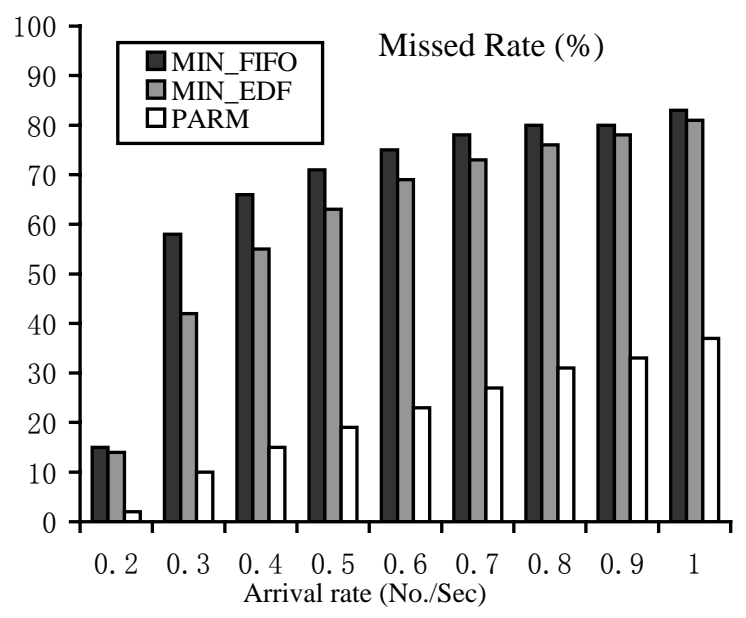

Figure 1. Missed rate vs. arrival rate Deadline range $=[100,500]$, data size $=[500,1000]$.

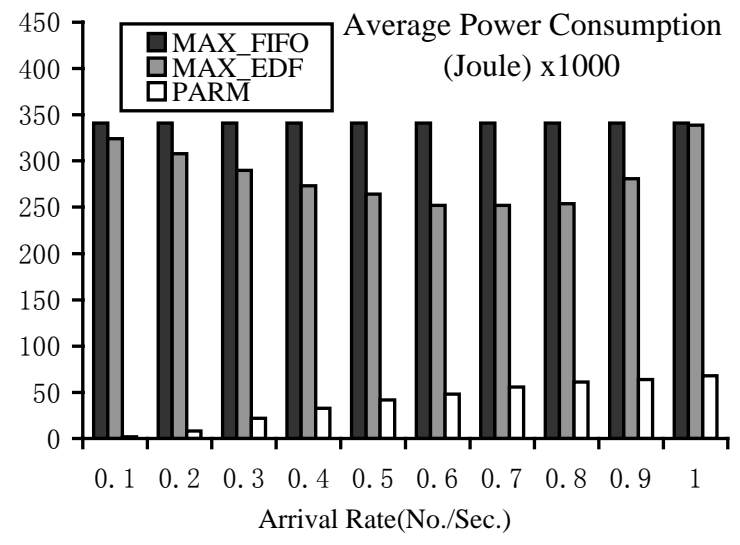

Figure 3. Average Power Consumption vs. arrival rate. Deadline rang $=[100,500]$, data $\operatorname{size}=[500,1000]$.

We observe from Figure 1 that MIN-EDF performs better than MIN-FIFO, and our PARM significant improves performance in terms of missed rate over both MIN-FIFO and MIN-EDF. This is mainly because MIN-FIFO and MIN-EDF assign the minimal transmission rate to admitted messages, resulting the maximal transmission time. Figure 1 illustrates that the missed rates of the three algorithms increase with the increasing value of arrival rate.

Figures 2 and 3 plot total and average power consumption of the AX-FIFO, MAX-EDF, and PARM when the deadline base is increased from 100 to 500 Seconds. Figures 2 and 3 reveal that PARM is much more energy-efficient than MAX-FIFO and MAX-EDF. We attribute the performance improvement of PARM to the fact that our scheme noticeably reduces energy consumption by judiciously assigning transmission rate for each admitted messages.

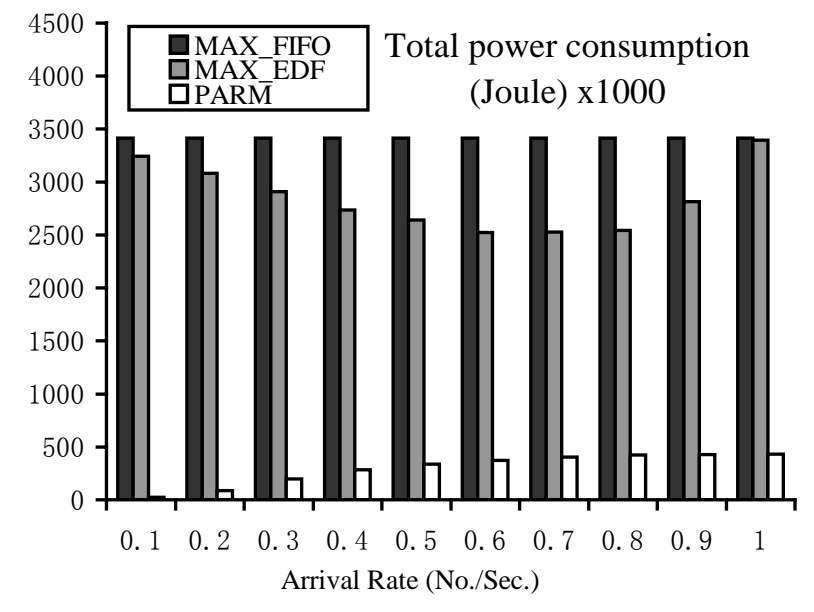

Figure 2. Total power consumption vs. arrival rate. Deadline rang $=[100,500]$, data size $=[500,100]$.

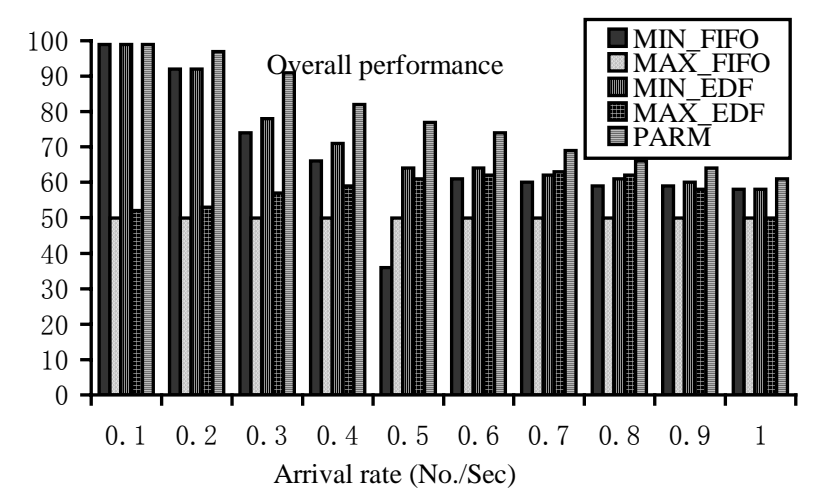

Figure 4. Overall performance vs. arrival rate. Deadline rang $=[100,500]$, data size $=[500,1000]$.

Figure 4 shows the overall performance of the five algorithms. It is observed from figure 4 that the PARM algorithm significantly outperforms all the other four alternatives. This can be explained by the fact that PARM considerably reduces energy consumption, while achieving lower missed rate. 


\subsection{Effect of deadlines}

Figures 5-8 show the impact of deadlines on the performance of real-time wireless networks. The observation drawn from Figure 5 is that the missed rates of the algorithms decrease with the increasing value of deadlines. This is mainly because loose deadlines enable the admission controller to accept more real-time messages to be transmitted in the wireless network.

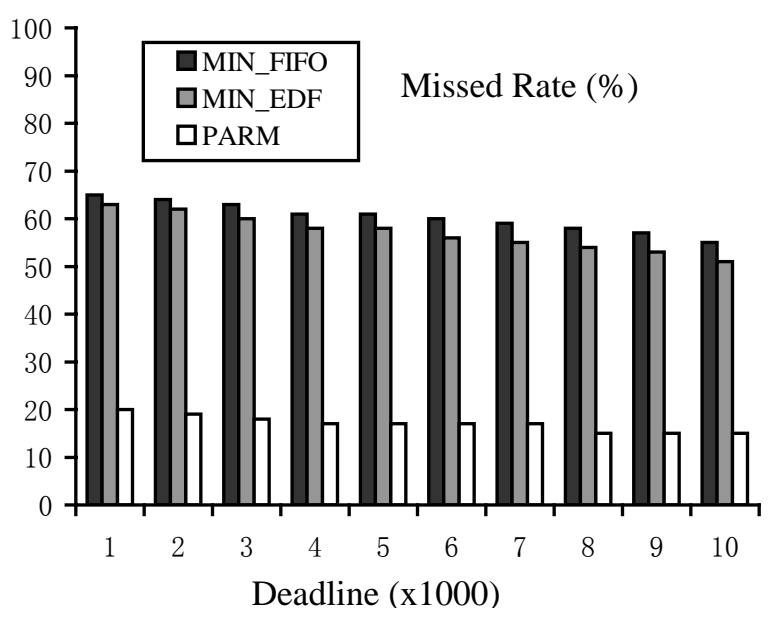

Figure 5. Missed rate vs. deadline. Arrival rate $=0.5$ No. $/$ Sec., data size $=[500,1000]$.

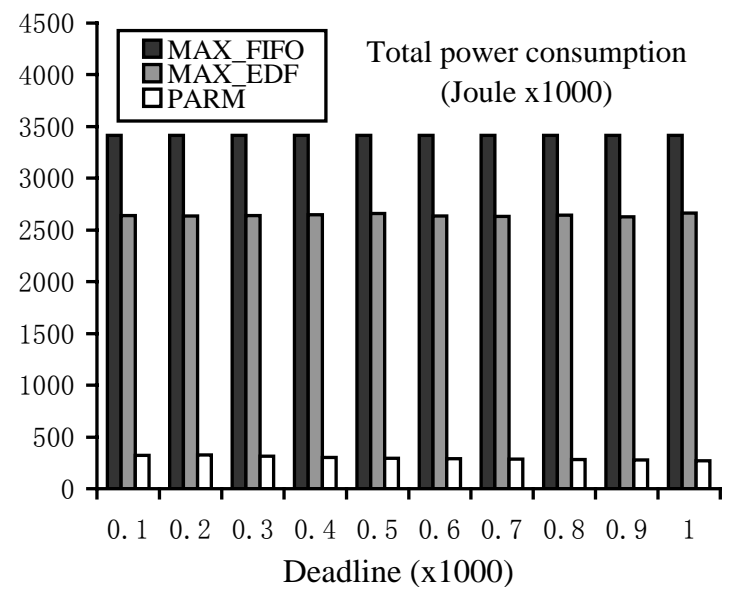

Figure 6. Total power consumption vs. deadline. Arrival rate $=0.5$ No. $/$ Sec., data size $=[500,1000]$.

Figures 6-7 demonstrate that the energy consumption of the three algorithms slighted decreases when deadlines increase. The implication of this result is that loose deadlines lead to relatively low energy consumption. The observation made from Figure 8 is that the PARM algorithm significantly outperforms all the other alternatives. This result is consistent with that plotted in Figure 4.

\section{Conclusions}

In this paper, we address the issue of scheduling real-time messages of multimedia applications in wireless networks subject to timing and power constraints. A power-aware real-time message scheduling heuristic, or PARM, is developed to generate optimal schedules for messages with deadline and power constraints. The PARM algorithm aims at minimizing energy consumption and the probability of missing deadlines for realtime messages transmitted in wireless networks. In particular, the proposed PARM scheme is energy efficient by the virtue of power-aware message scheduling for real-time messages. Before the presentation of PARM, we also proposed a power consumption model to quantitatively measure energy consumption based on message transmission rates. The model is used by the PARM scheme to calculate energy consumed by a real-time message transmitted in a particular rate.

To quantitatively evaluate the effectiveness and practicality of the proposed PARM scheme, we conducted extensive experiments using synthetic traces. Our experimental results show that PARM significantly improves the performance in terms of missed rate, energy consumption, and overall performance over four baseline schemes (MAX-FIFO, MIN-FIFO, MAX-EDF, MIN-EDF). The overall performance improvement of PARM is up to $61 \%$ over the four baseline algorithms.

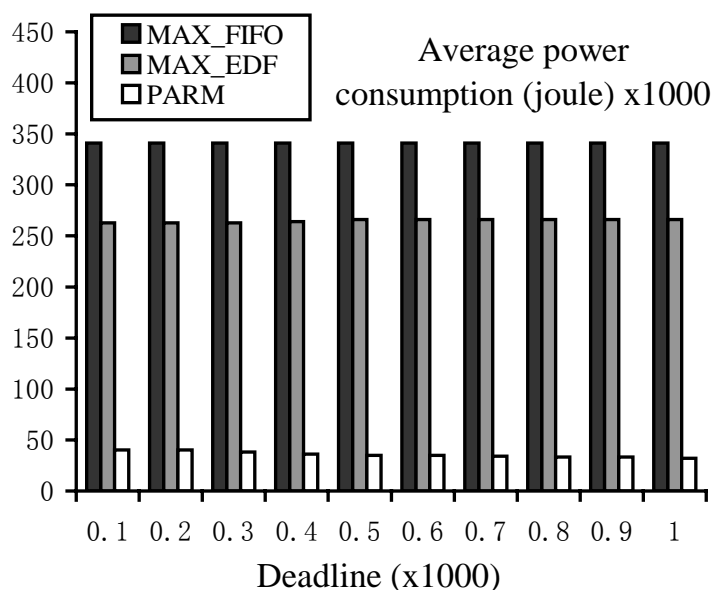

Figure 7. Average power consumption vs. deadline. Arrival Rate $=0.5$ No./Sec., data size $=[500,1000]$.

Future studies in this research can be performed in the following directions. First, we will extend our power-aware scheduling algorithm to deal with storage systems. Second, we plan to modify our message scheduling scheme to handle a large scale wireless network.

\section{Acknowledgements}

This work was partially supported by a research grant from the Intel Corporation and a start-up research fund (103295) from the Research and Economic Development Office of the New Mexico Institute of Mining and Technology. 


\section{References}

[1] X. Qin and H. Jiang, "Dynamic, Reliability-driven Scheduling of Parallel Real-time Jobs in Heterogeneous Systems," Proc. the 30th Int'l Conf. Parallel Processing, pp.113-122, Valencia, Spain, September 3-7, 2001.

[2] C. Jones, "A Survey of Energy Efficient Network Protocols for Wireless Networks, “ Wireless Networks 7, 343-358, 2001.

[3] C. L. Liu, J. W. Leyland, "Scheduling Algorithms for Multiprogramming in a Hard Real-Time Environment,” Journal of the ACM, Vol.20, No.1, pp.46-61, 1973.

[4] D. Clark, S. Shenker, L. Zhang, "Supporting Real-Time Applications in an Integrated Services Packet Network: Architecture and Mechanism," Proc. SIGCOMM, pp.14-26, 1992.

[5] G. Lipari, G, Buttazzo, "Scheduling Real-Time Multi-Task Applications in an Open system Real-Time Systems," Proceedings of the 11th Euro micro Conference on 9-11 June 1999 Page(s): 234 - 241.

[6] G. Manimaran and C. Siva Ram Murthy, “An Efficient Dynamic Scheduling Algorithm for Multiprocessor RealTime Systems. Parallel and Distributed Systems, IEEE Transactions on Volume 9, Issue 3, March 1998, pp. 312 319.

[7] J. A. Stine, G.Veciana, "Improving Energy Efficiency of Centrally Controlled Wireless Data Networks," Wireless Networks 8,681-700, 2002.

[8] J. A. Stankovic, M. Spuri, K. Ramamritham, G.C. buttazzo, Deadline Scheduling for real-time system - EDF and related Algorithms,” Kluer Academic Publishers, 1998.

[9] K. Ramamritham, J. A. Stankovic, "Dynamic task scheduling in distributed hard real-time system," IEEE Software, vol. 1, No. 3, July 1984.

[10] M. Havinga, G. Smit, "Energy-efficient wireless networking for multimedia Applications, "Wireless communications and Mobile Computing, Wily, 2001: pp.165-184.

[11] M. E. Thomadakis and J.-C. Liu, "On the efficient scheduling of non-periodic tasks in hard real-time systems,” Proc. $20^{\text {th }}$ IEEE Real-Time. Sys. Symp., pp.148151, 1999.

[12] P. Havinga, G. Smit, "Energy-efficient wireless networking for multimedia applications,” Wireless Communications and mobile Computing, Wiley, 2001: pp.165-184. 